\title{
Editorial:
}

\section{Células Dendríticas (CDs): Propiedades y limitaciones de su función inmunoterapéutica}

\author{
Gabriela Delgado M. \\ Bacterióloga, PhD en Ciencias Farmacéuticas, Docente de Microbiología, Directora del Grupo de Investigación en \\ Inmunotoxicología del Departamento de Farmacia. \\ Facultad de Ciencias \\ Universidad Nacional de Colombia
}

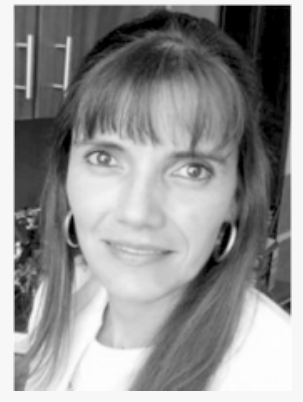

Hasta hace algunos años pensar en terapias celulares para el control de patologías de difícil control como el cáncer ó de enfermedades autoinmunes, era casi una utopía. No obstante los avances tecnológicos y los logros científicos han permitido que hoy en día el uso de «reactivos» celulares sea una realidad. El empleo de células madre ó células dendríticas (CDs) han mejorado la expectativa de vida de miles de personas en el mundo para quienes las terapias convencionales no se constituyen en una buena opción. Sin embargo, y hablando en términos de eficacia y seguridad de estas «formulaciones», es bastante lo que falta por conocer para lograr los resultados esperados.

En el caso particular del cáncer, considerado como un serio problema de salud pública en el mundo, el uso de inmunoterapia basada en el uso de CDs ha sido explorado con relativo éxito. Una de las principales razones que promovió el empleo de estas células, es la falla del sistema inmune para responder adecuadamente frente a los antígenos tumorales. Aprovechando la capacidad de las CDs como células presentadoras de antígeno (CPA) «profesionales» que activan eficientemente células tanto CD4+ como CD8+, se han diseñado múltiples protocolos clínicos, los cuales tienen como común denominador la diferenciación de CDs autólogas a partir de precursores mieloides o linfoides para su posterior autotransfusión al paciente. Una vez obtenidas, las CDs se pulsan con antígenos provenientes de distintas fuentes, como antígenos tumorales proteicos ó péptidos, antígenos administrados con productos microbianos utilizados como adyuvantes y CDs fusionadas con antígenos tumorales, entre otros.

La administración de vacunas basadas en CDs es tan variada como los protocolos clínicos en los cuales se han empleado. El protocolo óptimo, número de células, ruta y método de administración aún no se ha determinado, y corresponde más bien a los resultados clínicos y experimentales de casos particulares. Aunque el uso de la terapia celular ha favorecido el control de diversos tipos de cáncer, la dificultad para obtener tratamientos con mayor eficacia, obedece entre otros a la baja inmunogenicidad 
de algunos carcinomas que, además poseen una capacidad de evadir la respuesta inmune antitumoral efectiva, tanto in vitro como in vivo.

Más aún, cuando el sistema inmune logra ser activado, subsecuentemente puede ser modulado negativamente por antígenos tumorales, limitando de este modo, el resultado terapéutico de las CDs. El lograr revertir esta alteración, empleando moléculas solubles, por ejemplo citoquinas y anti-citoquinas, permitirá identificar factores que favorezcan una mejor respuesta in vivo, favoreciendo que CDs autólogas pulsadas con antígenos tumorales sean utilizadas como vacuna, en protocolos clínicos de inmunoterapia no convencional mucho más efectivos y seguros. En este proceso, se han evidenciado diversos mecanismos involucrados en la evasión de la respuesta inmune, algunos de ellos asociados con alteraciones funcionales de las CDs, y quizá sean los responsables de los fallos terapéuticos en algunos protocolos clínicos:

(i) $C D$ inmaduras (CDi): como ya se conoce, la co-estimulación es fundamental para la correcta activación de una respuesta celular $\mathrm{T}$ que induzca protección. En pacientes con cáncer renal y de próstata, se ha demostrado que aquellos tumores con rápido crecimiento contienen pequeñas cantidades de CDs y que estas usualmente presentan un fenotipo inmaduro, expresando muy pocas cantidades de moléculas co-estimuladoras como CD80 y CD86. Otros estudios han mostrado que CD maduras $(\mathrm{CDm})$ representan menos del 1\% intratumoral y cerca del 10\% peritumoral, y que dicho fenotipo no logra revertirse adicionando factor estimulador (GM-CSF) y factor de necrosis tumoral (TNF) ó CD40 ligando (citoquinas potentes inductoras de expresión de CD80 y CD86) sugiriendo que el defecto en estas células proviene desde la diferenciación a nivel de los progenitores hematopoyéticos. Las CDi no inducen una respuesta anti-tumoral, más aún ellas son capaces de generar tolerancia o anergia.

(ii) Células mieloides inmaduras (CMi): Son una población heterogénea de células mieloides que incluyen macrófagos, granulocitos, CDs y mieloides en estadios tempranos de diferenciación. Estas células, han sido ampliamente estudiadas en ratones y en humanos y se caracterizan por la expresión del marcador CD33 con una pérdida en la expresión de moléculas de clase II del CMH. La acumulación de éstas se asocia con una disminución de CDm circulantes en cáncer de cabeza y cuello, pulmón o seno, lo que conlleva a la inhibición de la respuesta inmune, asociada con la producción de grandes cantidades de reactivos oxigenados en presencia de antígenos tumorales, los cuales bloquean la actividad de linfocitos T CD8+ de forma específica.

(iii) Diferenciación anormal de células mieloides: Puede considerarse que el defecto a nivel de la actividad funcional de las CDs es sistémico y depende de factores solubles producidos por células tumorales. Esta idea fue confirmada al observar una diferenciación normal de CDs (desapereciendo de circulación las $\mathrm{CMi}$ ) una vez realizada la disección quirúrgica del tumor. 
(iv) Factor de crecimiento endotelial vascular (VEGF): Fue el primer factor derivado de tumores que mostró capacidad inhibitoria sobre CDs. y juega un papel fundamental en la formación de vasos durante la embriogénesis. Es producido por muchos tumores y participa en el desarrollo de la neovasculatura del tumor. Niveles aumentados de VEGF en plasma, se correlacionan con un pronóstico adverso de la neoplasia.

(v) Factor estimulador de colonias de macrófago (M-CSF) e interleuquina 6 (IL-6): A nivel experimental se ha demostrado que líneas celulares de carcinoma renal, liberan factores solubles que inhiben la diferenciación de progenitores CD34 a CDs, y que dicho efecto es revertido por el uso de anticuerpos neutralizantes de M-CSF e IL-6. Estudios adicionales han demostrado la participación de IL-6 como supresor de la maduración in vivo de CDs.

(vi) Factor estimulador de colonias granulocito-macrófago (GM-CSF): A pesar que cerca de un 30\% de líneas tumorales secretan esta molécula, su participación es importante en la maduración de CPA funcionales, al punto de ser un potencial terapéutico en vacunas contra cáncer. No obstante, se ha demostrado que grandes cantidades de GM-CSF van en detrimento del sistema inmune del individuo afectado con el tumor, mostrando un efecto negativo post-vacunación.

(vii) Inteleuquina 10 (IL-10): Su participación como modulador negativo de la respuesta inmune frente a tumores, radica en su capacidad de promover la diferenciación de CDi en tolerogénicas, a través de la pérdida ó disminución de los niveles de moléculas de co-estimulación. También se ha demostrado, que IL-10 bloquea la diferenciación de monocitos a $\mathrm{CD}$, e induce su maduración a macrófago. En resumen, la IL-10 puede afectar la diferenciación a CD en estadios tardíos.

(viii) Gangliósidos: Diversos tumores expresan patrones anormales de esta clase de glicoesfingolípidos ricos en ácido siálico cuando se comparan con tejido normal. La aparición de diferentes gangliósidos es útil en la determinación de la progresión de los tumores, ya que por ejemplo, gangliósidos derivados de melanoma muestran ser potentes inhibidores de la diferenciación de CDs.

A la luz de lo anterior, y con una visión optimista aunque realista, es mucho lo que se puede aportar en el conocimiento de las terapias celulares para hacer de estos productos farmacéuticos, formulaciones más eficaces y seguras. Con el concurso de todos, investigadores básicos y clínicos se facilitará el camino y aunque la disponibilidad de recursos pudiera llegar a ser una limitante en países como el nuestro, no puede constituirse en una barrera para generar conocimiento en torno a la terapia celular (células dendríticas y células madre) en cualquiera de sus dimensiones. Finalmente, a manera de epílogo filosófico y como una invitación a seguir trabajando con el espíritu perseverante que nos caracteriza les comparto lo que pienso: El subdesarrollo es en muchos casos producto de un auto-convencimiento mental que estamos obligados a vencer, si queremos que nos traten diferente. 